\title{
Empatía, inteligencia emocional y tolerancia a la diversidad en estudiantes de educación de una universidad pública peruana
}

\author{
Emotional intelligence, empathy and tolerance to the diversity in students \\ of education of a peruvian public university
}

\author{
Augusto Frisancho León ${ }^{1}$ \\ Universidad del Pacífico \\ Jaime Ramiro Aliaga Tovar ${ }^{2}$ \\ Universidad Nacional Mayor de San Marcos \\ Brian Norman Peña-Calero ${ }^{3}$ \\ Sociedad Peruana de Psicometría
}

\section{Resumen}

Los objetivos de la investigación fueron, en primer lugar, determinar los niveles de la empatía, inteligencia emocional y la tolerancia a la diversidad en una muestra conformada por 181 estudiantes de educación; en segundo lugar, establecer la relación existente entre las tres variables de estudio y, finalmente, determinar si hay diferencias en dichas variables debidas al sexo, el ciclo de estudios y las confesiones religiosas. Los instrumentos utilizados fueron el Trait Meta-Mood Scale-24, el Test de Empatía Cognitiva y Afectiva, y un instrumento de tolerancia a la diversidad. Los resultados a los que se arribaron indican la mayor parte de los estudiantes tiene un nivel alto de tolerancia a la diversidad y de empatía, y un nivel promedio de inteligencia emocional. Se confirmó la relación existente entre las puntuaciones de inteligencia emocional y empatía, así como las puntuaciones entre empatía y tolerancia a la diversidad, pero no entre inteligencia emocional y tolerancia a la diversidad. Asimismo, se encontró que hay diferencia en tolerancia a la diversidad y en empatía entre varones y mujeres, a favor de estas últimas; que no hay diferencia en función

1 Docente en Universidad del Pacífico.

Autor para correspondencia: afrisanchol@ulima.edu.pe ORCID: https://orcid.org/0000-0002-2394-523X

2 Docente Principal, Universidad Nacional Mayor de San Marcos. Lima, Perú.

E-mail: jaliagat@unmsm.edu.pe ORCID: https://orcid.org/0000-0002-4091-3205

3 Bachiller en Psicología por la Universidad Nacional Mayor de San Marcos. Lima, Perú. Miembro del Grupo de estudio Avances en Medición Psicológica. Consultor en Sociedad Peruana de Psicometría.

E-mail: brianmsm@gmail.com

(C) Los autores. Este artículo es publicado por la Revista de Investigación en Psicología de la Facultad de Psicología, Universidad Nacional Mayor de San Marcos. Este es un artículo de acceso abierto, distribuido bajo los términos de la licencia Creative Commons Atribucion - No Comercia_Compartir Igual 4.0 Internacional. (http://creativecommons.org/licenses/by-nc-sa/4.0/) que permite el uso no comercial, distribución y reproducción en cualquier medio, siempre que la obra original sea debidamente citada. 
al ciclo de estudios en ninguna de las variables estudiadas; y que los estudiantes que profesan la religión católica presentan un mayor nivel de empatía que los alumnos no creyentes.

Palabras clave: Inteligencia emocional; Tolerancia a la diversidad; Empatía, Educadores universitarios.

\begin{abstract}
The objectives of the research were, firstly, to determine the levels of emotional intelligence, empathy and tolerance to diversity in a sample of 181 students in education; secondly, to establish the relationship between the three study variables and, finally, to determine if there are differences in these variables due to sex, study cycle and religious denominations. The instruments used were the Meta-Mood Scale 24 Trait, the Cognitive and Affective Empathy Test, and a diversity tolerance instrument. The results arrived at indicate that most students have a high level of tolerance for diversity and empathy, and an average level of emotional intelligence. The relationship between scores of emotional intelligence and empathy was confirmed, as well as the scores between empathy and tolerance of diversity, but not between emotional intelligence and tolerance of diversity. Likewise, it was found that there is a difference in tolerance to diversity and in empathy between males and females, in favor of the latter; that there is no difference according to the cycle of studies in any of the variables studied; and that students who profess the Catholic religion present a higher level of empathy than non-believers.
\end{abstract}

Keywords: Emotional Intelligence; Tolerance to Diversity; Empathy, University Educators.

El adecuado desempeño de los educadores en una sociedad requiere de un gran compromiso sobre su actuar profesional, es decir, personas que además de una adecuada preparación en términos de didáctica y especialidad, también dispongan de aspectos psicológicos y emocionales que acompañen su labor como lo son niveles adecuados de la empatía, inteligencia emocional y la tolerancia a la diversidad. Este estudio pretende entender como estas cualidades-variables se encuentran en estudiantes de educación. Por otra parte, la investigación busca confirmar las relaciones señaladas entre la la empatía, inteligencia emocional y la tolerancia a la diversidad y, asimismo, determinar si la consideración de la variable sexo, ciclo de estudios y confesiones religiosas suponen una variación en las puntuaciones de las variables de interés.

De la revisión de la literatura sobre el tema, algunos autores como Aparicio, Cerda y Fernández (2015), sobre una muestra de 60 estudiantes de la Facultad de Medicina de la Universidad Popular Autónoma de Puebla, México, y Extremera y Fernández-Berrocal (2004) en una muestra de 184 estudiantes de la Facultad de Psicología de la Universidad de Málaga, España, indicaron que la inteligencia emocional y la empatía están relacionadas. Otros, como Esteban-Guitart, Rivas y Pérez (2012), en una muestra de 534 jóvenes de dos universidades públicas de Chiapas, México, han encontrado que la empatía y tolerancia a la diversidad se encuentran relacionadas, y una variación de la misma de acuerdo al sexo de la persona. Asimismo, también hay evidencia de que varones y mujeres difieren 
respecto a la inteligencia emocional, tal como lo demostraron Pérez y Castejón (2005) en una muestra de 250 alumnos provenientes de una institución española.

De acuerdo a lo señalado, se observa que las diversas investigaciones llevadas a cabo han establecido relaciones bivariadas existentes entre la empatía, inteligencia emocional y la tolerancia a la diversidad, sin embargo, ninguna de ellas ha considerado un análisis donde se involucre la interacción de las 3 variables en su conjunto, sinedo de especial importancia en el ámbito educativo.

\section{Objetivos}

- Establecer la magnitud de la relación entre la la empatía, inteligencia emocional y la tolerancia a la diversidad en estudiantes universitarios de una institución pública peruana.

- Determinar la función del sexo, ciclo de estudios y creencias religiosas con respecto a las variables de estudio central en universitarios de una institución pública peruana.

\section{Hipótesis de trabajo}

- Hay una correlación de magnitud mediana entre la la empatía, inteligencia emocional y la tolerancia a la diversidad en estudiantes de educación de una universidad pública peruana.

- La consideración del sexo, ciclo de estudios o creencias religiosas explica las variaciones en puntuaciones de la empatía, inteligencia emocional y la tolerancia a la diversidad entre los estudiantes de educación de una universidad pública peruana.

\section{MÉTODO}

\section{Diseño de investigación}

La presente es una investigación cuantitativa debido a que recolecta información numérica que es tratada a partir de análisis estadísticos, y de diseño no-experimental transversal correlacional así como descriptivo-comparativo (Hernández, Fernández y Baptista, 2014) de acuerdo a los objetivos propuestos. Las variables a correlacionar son la la empatía, inteligencia emocional y la tolerancia a la diversidad, mientras que las variables de comparación son el sexo, el ciclo de estudios y las creencias religiosas.

\section{Participantes}

Los participantes se eligieron mediante un muestreo no probabilístico intencional. La muestra fue de 181 estudiantes del 1er, 3er y 5to ciclo de estudios de la 
EAP de Educación de la UNMSM. La investigación se llevó a cabo durante el año 2017.

En la tabla 1 se observa que la muestra se encuentra conformada mayormente por mujeres y por estudiantes del primer ciclo. La tabla 2 muestra que la mayoría de los participantes profesa la religión católica.

\section{Tabla 1}

Distribución de la muestra por sexo y ciclo de estudios

\begin{tabular}{lccc}
\hline & Hombres & Mujeres & Total \\
\hline Primer ciclo & 29 & 49 & 78 \\
Tercer ciclo & 16 & 26 & 42 \\
Quinto ciclo & 21 & 40 & 61 \\
\hline Total & 66 & 115 & 181 \\
\hline
\end{tabular}

Tabla 2

Distribución de la muestra por creencia religiosa

\begin{tabular}{lcc}
\hline Creencia religiosa & $\mathbf{n}$ & $\mathbf{\%}$ \\
\hline Católicos & 87 & 48.7 \\
Cristianos no católicos & 43 & 23.76 \\
Otras religiones & 9 & 4.97 \\
No creyentes & 42 & 23.20 \\
\hline Total & 181 & 100.00 \\
\hline
\end{tabular}

\section{Técnicas e instrumentos}

Para evaluar la inteligencia emocional se recurrió a la Trait Meta-Mood Scale-24 (TMMS-24), de los autores Fernández-Berrocal, Extremera y Ramos (2004), la cual está basada en la TMMS-48 de Salovey, Mayer, Goldman, Turvey y Palfai (1995). Es una escala rasgo que evalúa el metaconocimiento de los estados emocionales; en concreto, las destrezas con las que podemos ser conscientes de nuestras propias emociones así como de nuestra capacidad para regularlas. Consta de tres dimensiones:

- Atención, referida a la capacidad de sentir y expresar los sentimientos de forma adecuada;

- Claridad, entendida como la capacidad de comprender bien los sentimientos propios;

- Regulación, que viene a ser la capacidad de regular los estados emocionales correctamente. 
La escala final está estructurada por 24 ítems (8 por cada dimensión). Los ítems presentan un escalamiento tipo Likert, que va desde 1 (Nada de acuerdo) hasta 5 (Totalmente de acuerdo). Su fiabilidad para cada componente es: Atención (0.90), Claridad (0.90), y Regulación (0.86). La fiabilidad test-retest presentada es adecuada (Fernández-Berrocal et al., 2004).

Para evaluar la empatía se utilizó el Test de Empatía Cognitiva y Afectiva (TECA), de López-Pérez, Fernández-Pinto y Abad (2008), compuesto por 33 ítems. El test presenta cuatro dimensiones: Adopción de perspectivas, comprensión emocional, estrés simpático, alegría empática.

Los ítems del test están organizados en una escala tipo Likert, que va desde 1 (Totalmente en desacuerdo) hasta 5 (Totalmente de acuerdo). La fiabilidad para el TECA global es de 0.86 y oscila entre 0.70 y 0.78 para las cuatro dimensiones. Asimismo, el TECA tiene validez convergente, pues presentó correlaciones de 0.63 con el Questionnaire Measure of Emotional Empathy (QMEE; Mehrabian y Epstein, 1972) y de 0.73 con la adaptación española del Interpersonal Reactivity Inventory (IRI; Pérez-Albéniz et al., 2003). Las puntuaciones altas en el test son indicativas de mayor nivel de empatía.

Finalmente, para medir la tolerancia a la diversidad se recurrió al Cuestionario de Tolerancia a la Diversidad de Lozano y Etxebarria (2007). Este instrumento está estructurado por 40 ítems, 34 de los cuales se distribuyen en cuatro subescalas, a los que se agregan 6 ítems adicionales con contenido referido al género, orientación sexual, entre otras variables. Las subescalas del cuestionario son: a) cultura, etnia, inmigración; b) características físicas e intelectuales; c) ideas políticas; d) pobreza, clase social

Los ítems del cuestionario están organizados en una escala tipo Likert, que parte desde 1 (Muy en desacuerdo) hasta la puntuación de 5 (Muy de acuerdo). La fiabilidad para la escala global es de 0.91 y oscila entre 0.66 y 0.85 para las cuatro subescalas (Lozano y Etxebarria, 2007). En el presente estudio se trabajó con un cuestionario de 30 ítems.

\section{Procesamiento y análisis de datos}

Previo a la comprobación de las hipótesis planteadas se verificó las propiedades psicométricas básicas (evidencias de validez y fiabilidad) de los instrumentos. Para brindar evidencia de validez basada en la estructura interna mediante el análisis factorial confirmatorio (AFC) se tuvo en consideración la naturaleza ordinal de los ítems, por lo que se utilizó la matriz de correlación policórica, y la evaluación del modelo especificado se efectuó mediante el estimador robusto WLSMV proporcionado por el paquete Lavaan 0.6.1 (Rosseel, 2012) del software libre R 3.5.0 ( $\mathrm{R}$ Core Team, 2017). La estimación de la fiabilidad mediante el método de consistencia interna se efectuó teniendo en cuenta la estructura factorial especificada 
y en consideración de la naturaleza ordinal de los ítems (matrices policóricas), mediante el uso de Omega de McDonalds (McDonald, 1999) obtenido mediante el paquete semTools 0.4.14 (semTools Contributors, 2016).

Posteriormente, se procedió a realizar la comprobación de los supuestos subyacentes al análisis de las hipótesis correlacionales, mediante la visualización de los datos (diagrama de dispersión) a fin de comprobar la linealidad de las puntuaciones y el análisis del ajuste normal univariado a la distribución de los datos mediante la prueba de Shapiro-Wilk. A partir de ello se decidió por una correlación de Pearson (si hay presencia de ajuste normal) o correlación de Spearman (si no se ajusta a la curva normal).

Por último, en el análisis de comparación de grupos se verificó, además del ajuste normal, la homocedasticidad de los datos entre los grupos a comparar decidiendo, para los grupos de dos categorías (sexo) la T de Student (ajuste normal y homocedasticidad), T con la corrección de Welch (ajuste normal y heterocedasticidad) o U de Mann Whitney (no ajuste normal). Mientras que para tres categorías o más (ciclos de estudio y creencias religiosas), se tiene los análisis de Anova de un factor (ajuste normal y homocedasticidad), Anova con la corrección de Welch (ajuste normal y heterocedasticidad) o Kruskal Wallis (no ajuste normal).

\section{RESULTADOS}

\section{Perfil de los sujetos en las variables de estudio}

A fin de conocer el perfil de los participantes en las variables de estudio se categorizó los puntajes de las pruebas mediante la estandarización teórica de los mismos a sus opciones de respuestas (Likert de 5 categorías).

En la tabla 3 se evidencia que, en cuanto a inteligencia emocional, el mayor porcentaje de los participantes, tanto varones como mujeres, se ubica en la categoría Promedio, y un porcentaje importante lo hace en la categoría Alto. Con respecto a la empatía, se observa que el mayor porcentaje de varones se ubica en la categoría Promedio, mientras el mayor porcentaje de mujeres lo hace en la categoría Alto. Y en cuanto a tolerancia a la diversidad, el mayor porcentaje de participantes, tanto varones como mujeres, se ubica en la categoría Alto, aunque se aprecia que un significativo porcentaje de mujeres se ubica en la categoría Muy Alto.

\section{Correlaciones entre las variables de estudio}

El análisis de normalidad de la distribución de los datos indica que las puntuaciones de inteligencia emocional $(\mathrm{S}-\mathrm{W}=0.99, \mathrm{p}=.216)$ y empatía $(\mathrm{S}-\mathrm{W}=0.99$, $\mathrm{p}=.329$ ) muestran un adecuado ajuste a la curva normal. En tanto, el análisis de 
tolerancia a la diversidad $(\mathrm{S}-\mathrm{W}=0.95, \mathrm{p}<.001)$ indica que no se ajusta a la distribución normal (ver Tabla 4).

Tabla 3

Distribución de los participantes acorde a las categorías de las variables

\begin{tabular}{|c|c|c|c|c|c|c|c|}
\hline & & \multicolumn{4}{|c|}{ Sexo } & \multirow{2}{*}{\multicolumn{2}{|c|}{ Total }} \\
\hline & & \multicolumn{2}{|c|}{ Varón } & \multicolumn{2}{|c|}{ Mujer } & & \\
\hline & & $\mathbf{n}$ & $\%$ & $\mathbf{n}$ & $\%$ & $\mathbf{n}$ & $\%$ \\
\hline \multirow{5}{*}{ Inteligencia emocional } & Muy Bajo & 0 & $0 \%$ & 0 & $0 \%$ & 0 & $0 \%$ \\
\hline & Bajo & 3 & $5 \%$ & 9 & $8 \%$ & 12 & $7 \%$ \\
\hline & Promedio & 38 & $58 \%$ & 65 & $57 \%$ & 103 & $57 \%$ \\
\hline & Alto & 21 & $32 \%$ & 38 & $33 \%$ & 59 & $33 \%$ \\
\hline & Muy Alto & 4 & $6 \%$ & 3 & $3 \%$ & 7 & $4 \%$ \\
\hline \multirow{5}{*}{ Empatía } & Muy Bajo & 0 & $0 \%$ & 0 & $0 \%$ & 0 & $0 \%$ \\
\hline & Bajo & 0 & $0 \%$ & 0 & $0 \%$ & 0 & $0 \%$ \\
\hline & Promedio & 45 & $68 \%$ & 29 & $25 \%$ & 74 & $41 \%$ \\
\hline & Alto & 21 & $32 \%$ & 82 & $71 \%$ & 103 & $57 \%$ \\
\hline & Muy Alto & 0 & $0 \%$ & 4 & $3 \%$ & 4 & $2 \%$ \\
\hline \multirow{5}{*}{ Tolerancia a la diversidad } & Muy Bajo & 0 & $0 \%$ & 0 & $0 \%$ & 0 & $0 \%$ \\
\hline & Bajo & 0 & $0 \%$ & 0 & $0 \%$ & 0 & $0 \%$ \\
\hline & Promedio & 5 & $8 \%$ & 5 & $4 \%$ & 10 & $6 \%$ \\
\hline & Alto & 49 & $74 \%$ & 61 & $53 \%$ & 110 & $61 \%$ \\
\hline & Muy Alto & 12 & $18 \%$ & 49 & $43 \%$ & 61 & $34 \%$ \\
\hline
\end{tabular}

Tabla 4

Estadisticos descriptivos y prueba de normalidad

\begin{tabular}{lccccc}
\hline & M & Mdn & DE & S-W & p \\
\hline Inteligencia emocional & 80.09 & 80.00 & 13.36 & 0.99 & .216 \\
Empatía & 119.23 & 119.00 & 12.70 & 0.99 & .329 \\
Tolerancia a la diversidad & 128.43 & 130.00 & 12.62 & 0.95 & $<.001$ \\
\hline
\end{tabular}

La inspección visual de las relaciones entre las variables se muestran en la Figura 1, donde se observa una tendencia lineal de los datos con mayor intensidad entre la variable empatía y tolerancia social.

La tabla 5 muestra el análisis de correlación entre las variables de estudio, donde se observa que existe correlación estadísticamente significativa, de tamaño pequeño, entre inteligencia emocional y empatía $(\mathrm{r}=.23, \mathrm{p}=.002)$, y de magnitud moderada entre empatía y tolerancia a la diversidad $(\rho=.48, \mathrm{p}<.001)$. No se observa correlación entre inteligencia emocional y tolerancia a la diversidad. 

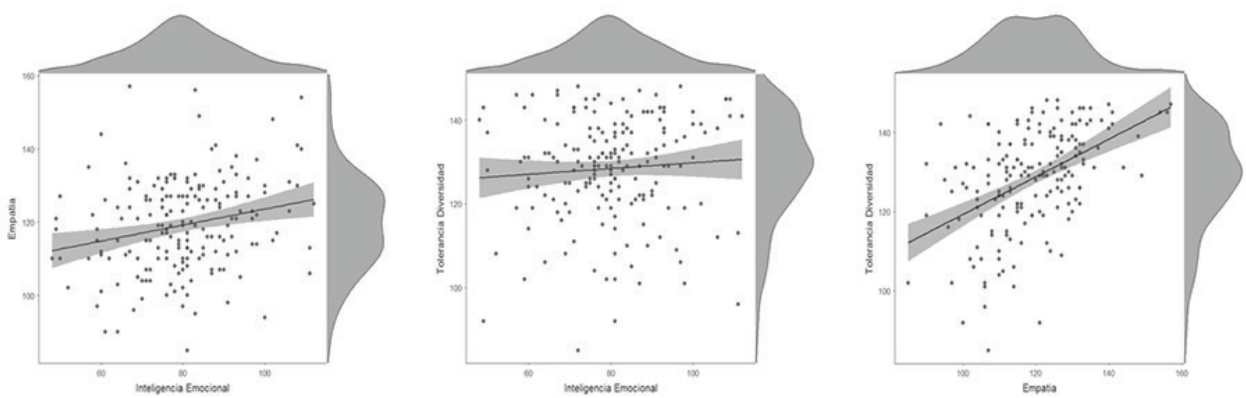

Figura 1. Diagrama de dispersión entre las variables de estudio y la distribución de sus datos.

Tabla 5

Correlaciones de las variables de estudio

\begin{tabular}{lcccc}
\hline & \multicolumn{2}{c}{ Empatía } & \multicolumn{2}{c}{ Tolerancia a la diversidad } \\
\cline { 2 - 6 } & $\mathbf{r}$ & $\mathbf{p}$ & $\boldsymbol{\rho}$ & $\mathbf{p}$ \\
\hline Inteligencia emocional & $\mathbf{0 . 2 3}$ & .002 & 0.08 & .297 \\
Empatía & & & $\mathbf{0 . 4 8}$ & $<.001$ \\
\hline
\end{tabular}

Nota. La correlación $r$ indica Pearson, mientras que $\rho$ indica Spearman.

Se muestra que existe correlación estadísticamente significativa, de tamaño pequeño, entre inteligencia emocional y empatía $(r=0.23, p=.002)$, y de magnitud moderada entre empatía y tolerancia a la diversidad $(\mathrm{r}=0.48, \mathrm{p}<.001)$. No existe correlación con significación estadística entre las puntuaciones de tolerancia a la diversidad y las de inteligencia emocional.

\section{Determinación de diferencias en función al sexo, ciclo de estudios y creen- cias religiosas}

En las puntuaciones de inteligencia emocional, se observa una diferencia descriptiva de 4 puntos a favor de los varones; sin embargo, en consideración al análisis inferencial, no implica una diferencia estadísticamente significativa. En cambio, en los puntajes de tolerancia a la diversidad y empatía sí se evidencian diferencias estadísticamente significativas, a favor de las mujeres, con una magnitud de grande y moderada, respectivamente (ver Tabla 6).

Con el fin de realizar la comparación de los puntajes con respecto al ciclo de estudios se realiza la comprobación de supuestos. En cuanto a la homocedasticidad, se observa que las puntuaciones de inteligencia emocional [Levene ${ }_{(2,178)}$ $=0.85, \mathrm{p}=.430]$ y de empatía [Levene $\left.{ }_{(2,178)}=1.63, \mathrm{p}=.199\right]$ se presentan con varianzas homogéneas en ambos grupos; en consecuencia, se procede a realizar el análisis de Anova de un factor sin ninguna corrección adicional. 
Tabla 6

Comparación en función del sexo

\begin{tabular}{|c|c|c|c|c|c|c|c|}
\hline & Sexo & M/Mdn & DE & $\mathbf{t}$ & $\mathbf{U}$ & $\mathbf{p}$ & d de Cohen \\
\hline \multirow{2}{*}{ Inteligencia emocional } & Varón & 82.15 & 13.52 & \multirow{2}{*}{1.58} & & \multirow{2}{*}{.117} & \multirow{2}{*}{0.24} \\
\hline & Mujer & 78.91 & 13.19 & & & & \\
\hline \multirow{2}{*}{ Empatía } & Varón & 112.38 & 11.17 & \multirow{2}{*}{-6.01} & & \multirow{2}{*}{$<.001$} & \multirow{2}{*}{0.93} \\
\hline & Mujer & 123.17 & 11.97 & & & & \\
\hline \multirow{2}{*}{ Tolerancia a la diversidad } & Varón & 125.50 & 12.06 & & \multirow{2}{*}{2451} & \multirow{2}{*}{$<.001$} & \multirow{2}{*}{0.58} \\
\hline & Mujer & 132.00 & 12.26 & & & & \\
\hline
\end{tabular}

Nota. Cuando se reporta el valor t de Student se indica el valor de la media en $\mathrm{M} / \mathrm{Mdn}$, mientras que cuando se reporte el valor de U de Mann Whitney se indica el valor de la mediana en M/Mdn.

El análisis de las variables en función del ciclo de estudios indica que las diferencias, a nivel descriptivo, encontradas en las puntuaciones de los diferentes ciclos académicos, no se evidencian a nivel inferencial de forma estadísticamente significativa (ver Tabla 7).

Tabla 7

Comparación en función del ciclo de estudios

\begin{tabular}{|c|c|c|c|c|c|c|}
\hline Variable & Ciclo & M/Mdn & DE & F & $\chi_{(2)}^{2}$ & $\mathbf{p}$ \\
\hline \multirow{3}{*}{ Inteligencia Emocional } & Primero & 79.08 & 12.13 & & & \\
\hline & Tercero & 79.36 & 14.84 & 0.85 & & .431 \\
\hline & Quinto & 81.90 & 13.83 & & & \\
\hline \multirow{3}{*}{ Empatía } & Primero & 120.15 & 11.55 & & & \\
\hline & Tercero & 118.31 & 12.04 & 0.37 & & .692 \\
\hline & Quinto & 118.69 & 14.55 & & & \\
\hline \multirow{3}{*}{ Tolerancia a la diversidad } & Primero & 130.00 & 10.56 & & & \\
\hline & Tercero & 125.50 & 13.21 & & 4.50 & .106 \\
\hline & Quinto & 131.00 & 14.16 & & & \\
\hline
\end{tabular}

Nota. Cuando se reporta el valor (F) se indica el valor de la media en M/Mdn, mientras que, cuando se reporte el valor de $\left(\chi^{2}\right)$ se indica el valor de la mediana en $\mathrm{M} / \mathrm{Mdn}$. El estadístico F es resultante de Anova de un factor, mientras que $\chi^{2}$ es parte del análisis por Kruskal Wallis.

Asimismo, para la comparación de los puntajes de las variables de estudios con respecto a las creencias religiosas, se tiene que el análisis de homocedasticidad de las varianzas de inteligencia emocional mostró que la variabilidad de los datos eran homogéneas [Levene ${ }_{(3,177)}=0.19, \mathrm{p}=.906$ ] al igual que en la variable

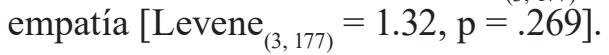

El análisis inferencial indica que existen diferencias con criterios de significancia estadśtica entre algunas de las medias de los puntajes de empatía en 
función de las creencias religiosas consideradas $(\mathrm{F}=4.04, \mathrm{p}=.008)$. Para poder observar específicamente entre quienes se produce esta diferencia, se realiza un análisis post-hoc de Tukey.

Tabla 8

Comparación en función de las creencias religiosas

\begin{tabular}{|c|c|c|c|c|c|c|}
\hline Variable & Creencias religiosas & M/Mdn & DE & $\mathbf{F}$ & $\chi_{(3)}^{2}$ & p \\
\hline \multirow{4}{*}{ Inteligencia emocional } & Católicos & 79.68 & 13.40 & \multirow{4}{*}{0.25} & & \multirow{4}{*}{.863} \\
\hline & Cristianos no católicos & 81.42 & 12.67 & & & \\
\hline & Otras religiones & 77.89 & 16.62 & & & \\
\hline & No creyentes & 80.07 & 13.63 & & & \\
\hline \multirow{4}{*}{ Empatía } & Católicos & 121.36 & 12.56 & \multirow{4}{*}{4.04} & & \multirow{4}{*}{.008} \\
\hline & Cristianos no católicos & 120.35 & 10.61 & & & \\
\hline & Otras religiones & 120.33 & 18.05 & & & \\
\hline & No creyentes & 113.45 & 12.39 & & & \\
\hline \multirow{4}{*}{$\begin{array}{l}\text { Tolerancia a la diver- } \\
\text { sidad }\end{array}$} & Católicos & 131.00 & 11.66 & & \multirow{4}{*}{3.72} & \multirow{4}{*}{.293} \\
\hline & Cristianos no católicos & 131.00 & 12.55 & & & \\
\hline & Otras religiones & 130.00 & 12.41 & & & \\
\hline & No creyentes & 125.50 & 14.32 & & & \\
\hline
\end{tabular}

Nota. El estadístico F es resultante de Anova de un factor, mientras que $\chi$ es parte el análisis por Kruskal Wallis.

El análisis post-hoc de Tukey, acerca de las puntuaciones de la variable empatía, indica que existen diferencias estadísticamente significativas entre los católicos y los no creyentes, con una diferencia de media de $7.91(\mathrm{p}=.005)$. Y aunque, el criterio de significancia estadística no indica la misma condición para cristianos no católicos y otras religiones, la consideración del tamaño del efecto entre sus diferencias los catalogan como variaciones grandes.

Tabla 9

Prueba HS Tukey de las puntuaciones de empatía

\begin{tabular}{lccc}
\hline Creencias religiosas & Diferencia de medias & $\mathrm{p}$ & $\mathrm{d}$ de Cohen \\
\hline Católicos - No creyentes & 7.91 & $\mathbf{. 0 0 5}$ & .64 \\
Cristiano no católicos - No creyentes & 6.90 & .054 & .56 \\
Otras religiones - No creyentes & 6.88 & .433 & .56 \\
\hline
\end{tabular}

\section{DISCUSIÓN}

La presente investigación encontró que los estudiantes de Educación evaluados muestran adecuados niveles de la empatía, inteligencia emocional y la tolerancia a la diversidad. 
Se corrobora la correlación estadísticamente significativa establecidad, de tamaño pequeño, entre inteligencia emocional y empatía. Esto es consecuente con lo hallado por Aparicio, Cerda y Fernández (2015) en México y por Extremera y Fernández-Berrocal (2004) en España. Esto indica que la conducta de entender y actuar desde los sentimientos, pensamientos y conductas de personas externas, se encuentra relacionado a la valoración de la inteligencia emocional o en su defecto es un componente del mismo. Así, acciones que promuevan la inteligencia emocional de la persona, también implicará cierto cambio en su empatía.

Además, la correlación entre la variable de tolerancia a la diversidad y la empatía fue estadísticamente significativa y de magnitud moderada, lo cual ha sido reportado también en otros estudios (Esteban-Guitart, Rivas y Pérez; 2012). Esto indica que las personas que tengan desarrollada una adecuada o alta empatía, muy probablemente evidenciarán conductas relacioandas a la tolerancia a la diversidad, esto es la tolerancia hacia culturas distintas, problemáticas personas y sociales.

Sin embargo, el análisis de correlación entre tolerancia a la diversidad y la inteligencia emocional no es estadísticamente significativa, además de tener un valor cercano a 0 . A partir de esto puede entenderse que a pesar de que la empatía es un factor medianamente importante cuando se valora la inteligencia emocional de una persona, no existe una relación directa entre tener alta empatía, alta inteligencia emocional y alta tolerancia a la diversidad. Las relaciones apareadas entre empatía-tolerancia a la diversidad y empatía-inteligencia emocional no implican una relación multivariada entre las variables señaladas. Para poder promover la tolerancia a la diversidad, será necesario trabajar en las personas ambos componentes: inteligencia emocional y empatía.

En cuanto a las diferencias en las variables estudiadas en función al sexo, se encontró que no existe diferencia estadísticamente significativa entre hombres y mujeres en inteligencia emocional, a diferencia de lo hallado por Pérez y Castejón (2005) en España. Es decir, las representaciones del constructo no parecen variar dependiendo de si alguien es hombre o mujer.

En cambio, en empatía y en tolerancia a la diversidad sí se encontraron diferencias estadísticamente significativas al considerar al sexo como variable diferenciadora, siendo esta variación a favor de las mujeres, con una magnitud grande en la primera y moderada en la segunda. Esto corrobora lo hallado por EstebanGuitart, Rivas y Pérez (2012) en México.

En cuanto a las diferencias en función al ciclo de estudios, no se encontraron diferencias estadísticamente significativas entre los alumnos del primer, tercer y quinto ciclo en ninguna de las variables estudiadas. Que el alumno avance en el ciclo académico universitario no está relacionado a un desarrollo espontáneo de estas variables. Es necesario que exista una política intencionada para lograr este 
cambio, a modo de intervenciones, talleres, programas o verbalizaciones dirigidas en el salón de clase.

Finalmente, en lo que se refiere a las diferencias en función a las creencias religiosas, se encontró que estas se dan en la variable empatía, pero sólo entre los estudiantes católicos y los estudiantes no creyentes. Puede argumentarse que las creencias y prácticas asociadas a la religión católica, orientan las conductas de las personas hacia aquellas que son considerdas como empáticas. Aunque pueda esperarse que una persona católica sea empática en comparación a una no-religiosa, existen situaciones en los que es al revés. En las otras variables (tolerancia a la diversidad e inteligencia emocional) no se hallaron diferencias estadísticamente significativas entre los participantes de las diferentes confesiones religiosas.

\section{CONCLUSIONES}

Los estudiantes de Educación evaluados muestran adecuados niveles de la empatía, inteligencia emocional y la tolerancia a la diversidad.

Existe correlación estadísticamente significativa, de tamaño pequeño, entre inteligencia emocional y empatía. Existe también una correlación estadísticamente significativa, de magnitud moderada, entre empatía y tolerancia a la diversidad. No se demostró que exista correlación entre inteligencia emocional y tolerancia a la diversidad.

Se encontraron diferencias estadísticamente significativas de acuerdo al sexo, a favor de estas últimas, en empatía y en tolerancia a la diversidad, más no en cuanto a inteligencia emocional.

No se encontraron diferencias estadísticamente significativas entre los alumnos del primer, tercer y quinto ciclo en ninguna de las variables estudiadas.

Se encontraron diferencias estadísticamente significativas entre los estudiantes católicos y los estudiantes no creyentes en la variable empatía, más no en las otras variables estudiadas.

\section{Fuentes de financiamiento / Funding:}

Recursos propios.

\section{Rol de los autores / Authors Roles:}

AFL: Formulación y desarrollo del proyecto de investigación.

JAT: Coautor del artículo con función de desarrollo teórico y conceptual del proyecto.

BPC: Análisis de datos y redacción de los mismos. 


\section{Aspectos éticos / legales; Ethics / legals:}

Los procedimientos de recolección de información y tratamiento de los datos se realizaron siguiendo las normativas de confidencialidad estipulados en los reglamentos genéricos de ética en investigación. Los participantes recibieron información acerca del sentido del estudio y se les indicó que su participación es enteramente voluntaria.

\section{Conflicto de intereses / Competing interests:}

Los autores declaran no tener conflictos de intereses relacionados al desarrollo de la presente investigación.

\section{REFERENCIAS}

Aparicio, E., Cerda, A. y Fernández, D. (2015). Inteligencia emocional y empatía en estudiantes de medicina de nuevo ingreso y pregrado. Integración Académica en Psicología, Vol. 3, Núm. 7, pp. 105-114.

Castillo, P. (2012). Nivel de empatía de los estudiantes practicantes de psicología clínica. (Tesis de Licenciatura). Universidad Rafael Landívar, Guatemala.

Esteban-Guitart, M., Rivas, M. y Pérez, M. (2011). Empatía y tolerancia a la diversidad en un contexto educativo intercultural. Universitas Psychologica, Vol. 11, Núm. 2, pp. 415-426.

Extremera, N. y Fernández-Berrocal, P. (2004). Inteligencia emocional, calidad de las relaciones interpersonales y empatía en estudiantes universitarios. Clínica y Salud, Vol. 15, Núm. 2, pp. 117-137.

Extremera, N, Fernández-Berrocal, P., Mestre, J. y Guil, R. (2004). Medidas de evaluación de la inteligencia emocional. Revista Latinoamericana de Psicología, Vol. 36, Núm. 2, pp. 209-228.

Fragoso-Luzuriaga, R. (2015). Inteligencia emocional y competencias emocionales en educación superior, ¿un mismo concepto? Revista Ibeoramericana de Educación Superior, Vol. VI, Núm. 16, pp. 110-125.

García, I. y Palomera, R. (2012). El desarrollo de la inteligencia emocional a través del teatro, para promover bienestar y respeto a la diversidad. Estilos de aprendizaje: investigaciones y experiencias. [V Congreso Mundial de Estilos de Aprendizaje], Santander, 27, 28 y 29 de junio de 2012.

Hernández, R., Fernández, C. y Baptista, P. (2014). Metodología de la investigación. México: McGraw-Hill.

Iacovella, J., Díaz-Lázaro, C. \& Richard's, M. (2015). Relación entre empatía y los cinco grandes factores de personalidad en una muestra de estudiantes universitarios. Revista Argentina de Ciencias del Comportamiento, 7(2), 14-21. 
Lozano y Etxebarria (2007). La tolerancia a la diversidad en los adolescentes y su relación con la autoestima, la empatía y el concepto del ser humano. Infancia y Aprendizaje, 30, (1), pp. 109-129.

Martí, J. (2011). Responsabilidad social universitaria: Estudio acerca de los comportamientos, los valores y la empatía en estudiantes de universidades iberoamericanas. (Tesis doctoral). Universidad de Valencia, España.

Martínez-Otero, V. (2011). La empatía en la educación: Estudio de una muestra de alumnos universitarios. Revista Electrónica de Psicología Iztacala, 14, (4), pp. 174-190.

Morales, F., Trianes, M. e Infante, L. (2013). Perfiles de valores éticos en estudiantes universitarios. Aula Abierta, Vol. 41, Núm. 2, pp. 55-66.

Pérez, N. y Castejón, J. (2005). Diferencias en inteligencia emocional en estudiantes universitarios. Análisis en función de la titulación y el género. Acta VIII Congreso Galaico Portugués de Psicopedagogía (1311-1321). Recuperado de http://www.educacion.udc.es/grupos/gipdae/documentos/congreso/VIIIcongreso/pdfs/165.pdf.

R Core Team (2018). R: A language and environment for statistical computing. R Foundation for Statistical Computing, Vienna, Austria. Recuperado de https://www.Rproject.org/.

SemTools Contributors (2016). SemTools: Useful tools for structural equation modeling. R package version 0.4-14. Recuperado de https://CRAN.R-project.org/ package $=$ semTools.

Ugarriza, N. y Pajares, L. (2005). La evaluación de la inteligencia emocional a través del inventario de BarOn ICE, en una muestra de niños y adolescentes. Persona, Núm. 8, pp. 11-58. 
ANEXO

TEST DE INTELIGENCIA EMOCIONAL (TMMS-24)

(Fernández-Berrocal, Extremera y Ramos, 2004)

Apellidos y nombres:

Sexo: ( ) Hombre ( ) Mujer Edad:

Especialidad:
( ) Inicial
( ) Primaria

( ) Secundaria - Biología y Química

( ) Secundaria - Matemática y Física

( ) Secundaria - Lengua, Literatura y Com.
Ciclo de estudios:

( ) Secundaria - Historia y Geografía

( ) Secundaria - Inglés y Castellano

( ) Secundaria - Filosofía, Tutoría y CCSS

Instrucciones:

A continuación encontrarás algunas afirmaciones sobre tus emociones y sentimientos. Lee atentamente cada frase e indica por favor el grado de acuerdo o desacuerdo con respecto a las mismas. Señala con una "X" la respuesta que más se aproxime a tus preferencias. No hay respuestas correctas o incorrectas, ni buenas o malas. No emplees mucho tiempo en cada respuesta.

\begin{tabular}{|c|c|c|c|c|}
\hline 1 & 2 & 3 & 4 & 5 \\
\hline $\begin{array}{l}\text { Nada de } \\
\text { acuerdo }\end{array}$ & $\begin{array}{c}\text { Algo de } \\
\text { acuerdo }\end{array}$ & $\begin{array}{c}\text { Bastante de } \\
\text { acuerdo }\end{array}$ & $\begin{array}{c}\text { Muy de } \\
\text { acuerdo }\end{array}$ & $\begin{array}{c}\text { Totalmente de } \\
\text { acuerdo }\end{array}$ \\
\hline
\end{tabular}

\begin{tabular}{|c|c|c|c|c|c|c|}
\hline & & 1 & 2 & 3 & 4 & 5 \\
\hline 1. & Presto mucha atención a los sentimientos. & & & & & \\
\hline 2. & Normalmente me preocupo mucho por lo que siento. & & & & & \\
\hline 3. & Normalmente dedico tiempo a pensar en mis emociones. & & & & & \\
\hline 4. & Pienso que merece la pena prestar atención a mis emociones y estados de ánimo. & & & & & \\
\hline 5. & Dejo que mis sentimientos afecten a mis pensamientos. & & & & & \\
\hline 6. & Pienso en mi estado de ánimo constantemente. & & & & & \\
\hline 7. & A menudo pienso en mis sentimientos. & & & & & \\
\hline 8. & Presto mucha atención a cómo me siento. & & & & & \\
\hline 9. & Tengo claros mis sentimientos. & & & & & \\
\hline 10. & Frecuentemente puedo definir mis sentimientos. & & & & & \\
\hline 11. & Casi siempre sé cómo me siento. & & & & & \\
\hline 12. & Normalmente conozco mis sentimientos sobre las personas. & & & & & \\
\hline 13. & A menudo me doy cuenta de mis sentimientos en diferentes situaciones. & & & & & \\
\hline 14. & Siempre puedo decir cómo me siento. & & & & & \\
\hline 15. & A veces puedo decir cuáles son mis emociones. & & & & & \\
\hline 16. & Puedo llegar a comprender mis sentimientos. & & & & & \\
\hline 17. & Aunque a veces me siento triste, suelo tener una visión optimista. & & & & & \\
\hline 18. & Aunque me sienta mal, procuro pensar en cosas agradables. & & & & & \\
\hline 19. & Cuando estoy triste, pienso en todos los placeres de la vida. & & & & & \\
\hline 20. & Intento tener pensamientos positivos aunque me sienta mal. & & & & & \\
\hline 21. & Si doy demasiadas vueltas a las cosas, complicándolas, trato de calmarme. & & & & & \\
\hline 22. & Me preocupo por tener un buen estado de ánimo., & & & & & \\
\hline 23. & Tengo mucha energía cuando me siento feliz. & & & & & \\
\hline 24. & Cuando estoy enfadado intento cambiar mi estado de ánimo. & & & & & \\
\hline
\end{tabular}

\begin{tabular}{|l|c|}
\hline \multicolumn{1}{|c|}{ Subescala } & PD \\
\hline Atención emocional & \\
\hline Claridad emocional & \\
\hline Reparación de emociones & \\
\hline
\end{tabular}




\section{TECA}

(López-Pérez, 2008)

Instrucciones:

A continuación encontrarás una serie de expresiones sobre las que nos gustaría que mostrases tu grado de acuerdo o desacuerdo.

\begin{tabular}{|c|c|c|c|c|}
\hline $\mathbf{1}$ & $\mathbf{2}$ & $\mathbf{3}$ & $\mathbf{4}$ & $\mathbf{5}$ \\
\hline $\begin{array}{c}\text { TOTALMENTE EN } \\
\text { DESACUERDO }\end{array}$ & EN & NI DE & DE & TOTALMENTE \\
DESACUERDO & $\begin{array}{c}\text { ACUERDO NI EN } \\
\text { DESACUERDO }\end{array}$ & ACUERDO & DE ACUERDO \\
\hline
\end{tabular}

1. Me resulta fácil darme cuenta de las intenciones de las personas que me rodean. $\begin{array}{lllll}1 & 2 & 3 & 4 & 5\end{array}$

2. Me siento bien si los demás se divierten (lo pasan bien).

$\begin{array}{lllll}1 & 2 & 3 & 4 & 5\end{array}$

3. Cuando un(a) amigo(a) está triste, yo también me pongo triste.

$\begin{array}{lllll}1 & 2 & 3 & 4 & 5\end{array}$

4. Si un(a) amigo(a) consigue un premio, me alegro mucho con él (ella).

$\begin{array}{lllll}1 & 2 & 3 & 4 & 5\end{array}$

5. Si veo que alguien ha tenido un accidente, me pongo triste.

$\begin{array}{lllll}1 & 2 & 3 & 4 & 5\end{array}$

6. Antes de tomar una decisión, intento tener en cuenta las opiniones de las personas cercanas a mí.

$\begin{array}{lllll}1 & 2 & 3 & 4 & 5\end{array}$

7. Rara vez reconozco cómo se siente una persona cercana a mí con sólo mirarla.

$\begin{array}{lllll}1 & 2 & 3 & 4 & 5\end{array}$

8. Me afectan poco las cosas malas que les suceden a las chicas y chicos de otros pueblos.

$\begin{array}{lllll}1 & 2 & 3 & 4 & 5\end{array}$

9. Me alegra ver que un amigo nuevo se encuentra a gusto en nuestro grupo.

$\begin{array}{lllll}1 & 2 & 3 & 4 & 5\end{array}$

10. Me cuesta entender cómo se siente otra persona ante una situación nueva para mí.

$\begin{array}{lllll}1 & 2 & 3 & 4 & 5\end{array}$

11. Cuando un(a) amigo(a) se ha portado mal conmigo, intento entender los motivos por los que lo ha hecho.

12. Me cuesta llorar con lo que les sucede a otros.

$\begin{array}{lllll}1 & 2 & 3 & 4 & 5\end{array}$

13. Reconozco fácilmente cuándo alguien cercano está de mal humor.

14. Pocas veces me doy cuenta cuándo la persona que tengo al lado se siente mal.

$\begin{array}{lllll}1 & 2 & 3 & 4 & 5\end{array}$

15. Frente a una situación, intento ponerme en el lugar de las personas cercanas a mí para saber cómo actuarán.

16. Cuando a una persona le sucede algo bueno, siento alegría.

$\begin{array}{lllll}1 & 2 & 3 & 4 & 5\end{array}$

17. Si tengo mi opinión clara, presto poca atención a la opinión de los demás.

$\begin{array}{lllll}1 & 2 & 3 & 4 & 5\end{array}$

18. A veces sufro mucho con las cosas malas que les suceden a otros chicos o chicas.

$\begin{array}{lllll}1 & 2 & 3 & 4 & 5\end{array}$

19. Me siento feliz al ver felices a otras personas.

$\begin{array}{lllll}1 & 2 & 3 & 4 & 5\end{array}$

$\begin{array}{lllll}1 & 2 & 3 & 4 & 5\end{array}$

20. Cuando alguien tiene un problema, intento pensar cómo me sentiría si estuviera en su lugar.

21. Me alegro poco cuando una persona me cuenta que ha tenido buena suerte.

22. Cuando veo que una persona ha recibido un regalo, me pongo alegre.

$\begin{array}{lllll}1 & 2 & 3 & 4 & 5\end{array}$

$\begin{array}{lllll}1 & 2 & 3 & 4 & 5\end{array}$

23. Lloro fácilmente cuando escucho las cosas tristes que les han sucedido a desconocidos. $\quad \begin{array}{llllll}1 & 2 & 3 & 4 & 5\end{array}$

24. Cuando conozco gente nueva me doy cuenta enseguida de lo que piensan de mí.

25. Le doy poca importancia a que mis amigos(as) estén bien.

26. Me resulta difícil ponerme en el lugar de otras personas, para ver las cosas como ellas.

27. Entender cómo se siente alguien cercano es algo muy fácil para mí.

$\begin{array}{lllll}1 & 2 & 3 & 4 & 5\end{array}$

$\begin{array}{lllll}1 & 2 & 3 & 4 & 5\end{array}$

$\begin{array}{lllll}1 & 2 & 3 & 4 & 5\end{array}$

28. Muy pocas veces me pongo triste con los problemas de otros chicos y chicas.

29. Intento comprender mejor a mis amigos(as) poniéndome en su lugar.

$\begin{array}{lllll}1 & 2 & 3 & 4 & 5\end{array}$

$\begin{array}{lllll}1 & 2 & 3 & 4 & 5\end{array}$

30. Creo que soy una persona fría y de pocos sentimientos, porque no me emociono fácilmente. $\begin{array}{lllllll}1 & 2 & 3 & 4 & 5\end{array}$

31. Me doy cuenta cuando las personas cercanas a mí están contentas, aunque no me hayan contado el motivo.

$\begin{array}{lllll}1 & 2 & 3 & 4 & 5\end{array}$

$\begin{array}{lllll}1 & 2 & 3 & 4 & 5\end{array}$

32. Cuando no estoy de acuerdo con un(a) amigo(a), me resulta difícil entender su punto de vista.

$\begin{array}{lllll}1 & 2 & 3 & 4 & 5\end{array}$

$\begin{array}{lllll}1 & 2 & 3 & 4 & 5\end{array}$

33. Me doy cuenta cuando alguien cercano intenta esconder sus verdaderos sentimientos.

$\begin{array}{lllll}1 & 2 & 3 & 4 & 5\end{array}$

$\begin{array}{lllll}1 & 2 & 3 & 4 & 5\end{array}$

$\begin{array}{lllll}1 & 2 & 3 & 4 & 5\end{array}$ 

peruana

\title{
CUESTIONARIO TD
}

\author{
(Lozano y Etxebarria, 2003)
}

Instrucciones:

A continuación encontrarás una serie de expresiones sobre las que nos gustaría que mostrases tu grado de acuerdo o desacuerdo.

\begin{tabular}{|c|c|c|c|c|}
\hline $\mathbf{1}$ & $\mathbf{2}$ & $\mathbf{3}$ & $\mathbf{4}$ & $\mathbf{5}$ \\
\hline $\begin{array}{c}\text { MUY EN } \\
\text { DESACUERDO }\end{array}$ & $\begin{array}{c}\text { ALGO EN } \\
\text { DESACUERDO }\end{array}$ & $\begin{array}{c}\text { NI DE } \\
\text { ACUERDO NI EN } \\
\text { DESACUERDO }\end{array}$ & $\begin{array}{c}\text { ALGO DE } \\
\text { ACUERDO }\end{array}$ & $\begin{array}{c}\text { MUY DE } \\
\text { ACUERDO }\end{array}$ \\
\hline
\end{tabular}

1. Me agrada que en mi ciudad residan personas procedentes de las diferentes regiones del país. $\quad \begin{array}{llllll}1 & 2 & 3 & 4 & 5\end{array}$

2. Me preocupan las dificultades de aprendizaje que presentan ciertos compañeros(as) y estoy dispuesto a ayudarles.

3. Suelo burlarme de los defectos físicos de los demás.

$\begin{array}{lllll}1 & 2 & 3 & 4 & 5\end{array}$

4. Me molesta que la gente exprese opiniones negativas sobre otras personas o grupos, sin apenas conocerlos, por puro prejuicio.

5. Me cuesta aceptar a las personas con tendencias homosexuales.

$\begin{array}{lllll}1 & 2 & 3 & 4 & 5\end{array}$

6. Todas las personas son igualmente valiosas: es tan valiosa la persona que dirige la empresa como la que la limpia.

7. Prefiero no relacionarme con personas de otras etnias y/o culturas

$\begin{array}{lllll}1 & 2 & 3 & 4 & 5\end{array}$

8. Tiendo a tratar de la misma forma al pobre que al rico.

$\begin{array}{lllll}1 & 2 & 3 & 4 & 5\end{array}$

9. Me parece bien tener en mi clase a compañeros(as) con discapacidad motriz porque todas las personas tenemos los mismos derechos.

$\begin{array}{lllll}1 & 2 & 3 & 4 & 5\end{array}$

10. Suelo respetar a las personas que en cuestiones políticas piensan de un modo distinto a mí. $\begin{array}{llllll}1 & 2 & 3 & 4 & 5\end{array}$ $\begin{aligned} & \text { 11. No suele afectarme que alguien menosprecie a un compañero(a) que tenga algún tipo de } \\ & \text { deficiencia. }\end{aligned}$

$\begin{array}{lllll}1 & 2 & 3 & 4 & 5\end{array}$

$\begin{array}{lllll}1 & 2 & 3 & 4 & 5\end{array}$

12. Siento cierta incomodidad con personas que proceden de otros lugares (de la Sierra, de la Selva...).

\begin{tabular}{|c|c|c|c|c|c|}
\hline 13. Considero que las personas que no piensan como yo están equivocadas. & 1 & 2 & 3 & 4 & r \\
\hline 14. Mi mejor amigo(a) podría ser una persona de otra raza o etnia. & 1 & 2 & 3 & 4 & 5 \\
\hline 15. Las personas con deficiencias no deberían asistir a clases normales & 1 & 2 & 3 & 4 & \\
\hline $\begin{array}{l}\text { 16. Es bueno que en este país haya políticos con distintos puntos de vista y distintas soluciones } \\
\text { para los problemas existentes. }\end{array}$ & 1 & 2 & 3 & 4 & \\
\hline 17. Chicos y chicas podemos aprender mucho unos de otros. & 1 & 2 & 3 & 4 & 5 \\
\hline 18. Yo suelo pensar: "Tanto tienes, tanto vales". & 1 & 2 & 3 & 4 & 5 \\
\hline $\begin{array}{l}\text { 19. Entiendo que las personas que viven en situac } \\
\text { si yo estuviese en su lugar haría lo mismo para so }\end{array}$ & 1 & 2 & 3 & 4 & \\
\hline 20. No suelo sentir demasiada simpatía por las personas de una cultura diferente a la mía. & 1 & 2 & 3 & 4 & 5 \\
\hline $\begin{array}{l}\text { 21. Tener a compañeros(as) con necesidades educativas especiales en las aulas es positivo para } \\
\text { todos. }\end{array}$ & 1 & 2 & 3 & 4 & \\
\hline 22. No me gustan las personas que tienen ideas diferentes a las mías. & 1 & 2 & 3 & 4 & 5 \\
\hline 23. Suelo mirar por encima del hombro a aquellas personas que carecen de recursos económicos. & 1 & 2 & 3 & 4 & 5 \\
\hline $\begin{array}{l}\text { 24. Puesto que todo el mundo tiene derecho a opinar, suelo ser tolerante con las ideas de los } \\
\text { demás, aunque no las comparta. }\end{array}$ & 1 & 2 & 3 & 4 & \\
\hline 25. Me siento incómodo(a) cuando estoy con personas que son muy distintas a mí. & 1 & 2 & 3 & 4 & 5 \\
\hline 26. No me importaría tener un amigo o amiga homosexual. & 1 & 2 & 3 & 4 & 5 \\
\hline 27. Prefiero relacionarme con personas que poseen dinero y visten bien. & 1 & 2 & 3 & 4 & 5 \\
\hline $\begin{array}{l}\text { 28. Si los chicos y chicas nos relacionamos entre nosotros, cuando seamos hombres y mujeres } \\
\text { adultos(as) habrá menores diferencias sociales entre los dos sexos. }\end{array}$ & 1 & 2 & 3 & 4 & \\
\hline 29. Me molesta el tipo de prensa que se aleja de mi ideología política. & 1 & 2 & 3 & 4 & 5 \\
\hline 30. Me describiría a mí mismo(a) como una persona poco tolerante. & 1 & 2 & 3 & 4 & \\
\hline
\end{tabular}


